\title{
Indications for total-body computed tomography in blunt trauma patients: a systematic review
}

\author{
K. Treskes ${ }^{1}$ T. P. Saltzherr ${ }^{1}$ J. S. K. Luitse ${ }^{1}$ L. F. M. Beenen ${ }^{2}$ J. C. Goslings ${ }^{1}$
}

Received: 16 December 2015 / Accepted: 12 July 2016 / Published online: 19 July 2016

(C) The Author(s) 2016. This article is published with open access at Springerlink.com

\begin{abstract}
Purpose Total-body CT scanning (TBCT) could improve the initial in-hospital evaluation of severe trauma patients. Indications for TBCT, however, differ between trauma centers, so more insight in how to select patients that could benefit from TBCT is required. The aim of this review was to give an overview of currently used indications for totalbody $\mathrm{CT}$ in trauma patients and to describe mortality and Injury Severity Scores of patient groups selected for TBCT. Methods A systematic review was performed by searching MEDLINE and Embase databases. Studies evaluating or describing criteria for selection of patients with potentially severe injuries for TBCT during initial trauma care were included. Also, studies comparing total-body CT during the initial assessment of injured patients with conventional imaging and selective $\mathrm{CT}$ in specific patient groups were included.

Results Thirty eligible studies were identified. Three studies evaluated indications for TBCT in trauma with divergent methods. Combinations of compromised vital parameters, severe trauma mechanisms and clinical suspicion on severe injuries are often used indications; however, clinical
\end{abstract}

Electronic supplementary material The online version of this article (doi:10.1007/s00068-016-0711-4) contains supplementary material, which is available to authorized users.

K. Treskes

k.treskes@amc.nl

1 Trauma Unit, Department of Surgery, Academic Medical Center, Meibergdreef 9, 1105 AZ Amsterdam, The Netherlands

2 Department of Radiology, Academic Medical Center, Amsterdam, Meibergdreef 9, 1105 AZ Amsterdam, The Netherlands judgement is used as well. Studies describing TBCT indications selected patients in different ways and were difficult to compare regarding mortality and injury severity. Conclusions Indications for TBCT in trauma show a wide variety in structure and cut-off values for vital parameters and trauma mechanism dimensions. Consensus on indications for TBCT in trauma is lacking.

Keywords Total-body CT · Whole body imaging · Multiple trauma $\cdot$ Wounds and injuries · Computed tomography

\section{Introduction}

The work-up of trauma patients by ATLS (advanced trauma life support) guidelines uses a step-up approach for diagnostic imaging. After conventional radiography of the chest and pelvis and focused assessment by sonography (FAST), selective computed tomography can be performed subsequently on indication [1]. Ongoing improvements in speed and accuracy of computed tomography (CT) and increased availability of $\mathrm{CT}$ scanners in or nearby the trauma room made immediate total-body CT (TBCT) feasible as a diagnostic tool in the initial assessment of trauma patients. Initial trauma care, thus, might be improved when total-body CT scan is incorporated in the initial assessment of a potentially multiple and severely injured patient [2].

A disadvantage of TBCT scanning is increased radiation exposure for patients that appear to have minor injuries for which selective CT scanning on indication could be sufficient. For the overall group of trauma patients, the proportion of patients receiving a high radiation dose of $>20 \mathrm{mSv}$ at the trauma room is increased [3]. For multitrauma patients, the radiation dose is, however, comparable for 
the complete hospital admission [4]. To prevent excessive radiation exposure, the appropriate selection of patients for TBCT is essential $[3,5]$. The decision to perform an immediate TBCT is based on information obtained during the pre-hospital phase and the first in-hospital assessment. Therefore, indications such as compromised vital parameters, clinical suspicion on severe injuries and high-risk injury mechanisms are often used to select trauma patients that might benefit from immediate TBCT.

Justification for performing a TBCT is only possible in hindsight, when all diagnoses have been confirmed by radiologic imaging, interventions and the clinical course. Moreover, different outcome measures are used to justify TBCT, such as: classification as multiple or severely injured patient by anatomical scoring systems (e.g., Injury Severity Score) or certain high-risk profiles for injuries [6-8]. To improve selection and to guide future research on the proper indications for TBCT after major trauma, a better insight in current indications is required. Therefore, the aim of this review was (1) to give an overview of currently used indications for total-body CT in trauma patients and (2) to describe mortality and Injury Severity Scores of patient groups selected for TBCT.

\section{Methods}

For this systematic review, the preferred reporting items for systematic reviews and meta-analyses (PRISMA) are used as a guideline [9].

\section{Inclusion and exclusion criteria}

Studies evaluating or describing indications for TBCT during initial trauma care were included. Also, studies comparing TBCT during the initial assessment of injured patients with conventional imaging and selective $\mathrm{CT}$ in specific patient groups were included. TBCT should at least comprise the following body regions: head, neck, thorax, abdomen and pelvis. For selection of studies, no distinction was made between immediate TBCT and TBCT with preceding conventional radiologic imaging. Reviews, randomized and observational studies describing original data were eligible for inclusion. Study protocols, case reports and editorials were excluded. Literature in a language other than English or German was also excluded.

\section{Search strategy}

The MEDLINE and Embase library databases were searched for articles published between 1947 and July 2014. The search terms consisted of synonyms of 'total-body CT' combined with synonyms and words related to trauma and injury. The full search is presented in Supplementary
Appendix 2. The last search was performed in July 2014 and was conducted with the help of a clinical librarian. A crossreference search was performed on the included articles.

\section{Study selection and data extraction}

Two reviewers independently assessed titles and abstracts of all studies identified by the initial search and excluded irrelevant studies. Second, the full texts of the remaining eligible studies were assessed to determine whether they met the inclusion criteria. Any discrepancies in inclusion were resolved by discussion between the reviewers. In case no consensus was reached, this was solved by a third reviewer. The following data from each included paper were extracted: author, publication year, country, study design, inclusion criteria, sample size, Injury Severity Score (ISS), indications for TBCT, and outcome.

\section{Results}

\section{Study selection}

The search identified 532 records from the MEDLINE database and 1006 records from the Embase database. 366 duplicates were removed. 30 studies were included for data extraction (Fig. 1). Included study designs were retrospective for 17 studies and prospective or observational for 10 studies. The remaining three were a randomized clinical trial, a case-matched study and one questionnaire survey. Studies were published between 2003 and 2013, except for one, which was published in 1998.

\section{Studies on TBCT indications}

For three included studies, the main objective was to evaluate indications for TBCT in trauma patients. Wurmb et al. [8] assessed whether a triage scheme could appropriately select sedated and ventilated patients with severe trauma for TBCT scanning. This triage scheme used specific trauma mechanisms, compromised vital signs and clinically obvious injuries. An Injury Severity Score (ISS) of 16 or higher was used to define severe trauma. Sensitivity of this triage scheme for severe trauma was $96.7 \%$ and positive predictive value was $69.4 \%$.

Hsiao et al. [7] also used an anatomical definition of severe trauma to justify TBCT for patients that triggered trauma team activation and were CT-scanned during the initial in-hospital assessment. An Abbreviated Injury Score (AIS) of 2 or more in two or more body regions defined multi-regional injury. Clinical judgement had a sensitivity of $50 \%$ and a $32 \%$ positive predictive value for multiregion injury. Mean ISS was 17 (SD16) for patients that 
Fig. 1 Flowchart for the selection of studies

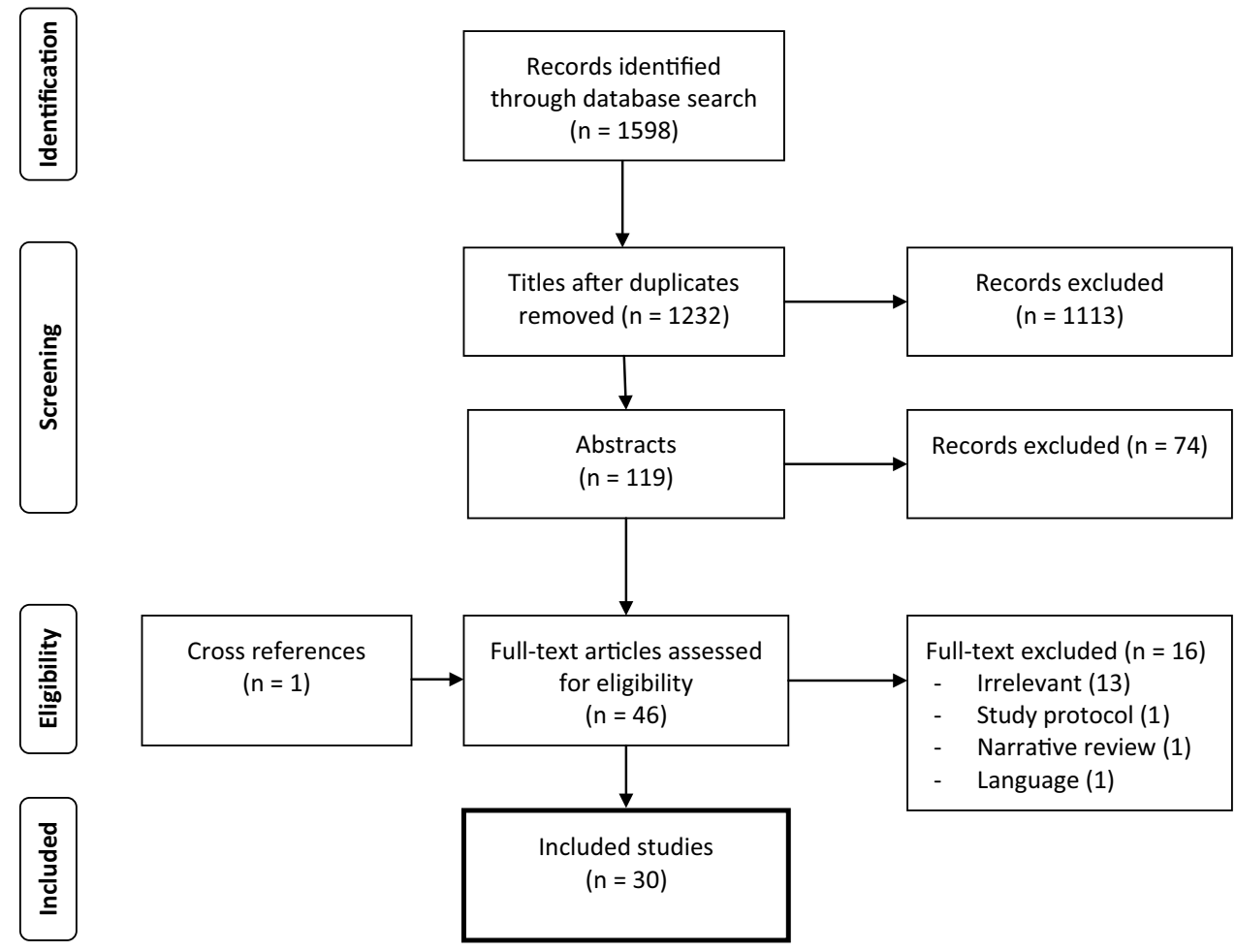

Table 2 shows that selection of multitrauma patients was often a result of the study design rather than selection of patients for TBCT by trauma leaders. Five retrospective studies enrolled patients with an ISS of 16 or higher [2, 11-14]. Weninger et al. [15] included only patients with an ISS of 17 or higher and at least one body region with an AIS of 4 or higher. Rieger et al. [16] included patients with an ISS of 18 or higher. Two prospective studies included patients who triggered trauma team activation and reported a median ISS of 5 (IQR 1-14) and 13 [1-17] for patients who underwent TBCT based on clinical judgement [17, 18]. Hsiao et al. [7] retrospectively selected patients receiving CT imaging during trauma assessment and reported a mean ISS of 17 (SD16) for patients with an indication for TBCT by clinical judgement. The remaining studies that described an indication by clinical judgement, retrospectively selected patients by ISS or bleeding control measures (Table 2).

In the appendix, the described TBCT indications after trauma and cut-off values for vital parameters and trauma mechanism dimensions are presented from 30 included articles. These are categorized by vital parameters, clinical suspicious injuries, high-risk trauma mechanism and contraindications. For all the included literature, minor age and isolated penetrating injury were formulated as contraindications for TBCT or indirectly formulated by including only adult patients sustaining blunt trauma. 
Table 1 Overview of included studies

\begin{tabular}{|c|c|c|c|c|c|}
\hline Author, study year, country & $\begin{array}{l}\text { Study } \\
\text { design }\end{array}$ & $\begin{array}{l}\text { Patients overall } \\
\text { (TBCT) }\end{array}$ & $\begin{array}{l}\text { ISS, median (IQR) TBCT/ } \\
\text { control/overall }\end{array}$ & TBCT indications & Outcome \\
\hline Sierink 2014, The Netherlands [14] & $\mathrm{CM}$ & $304(152)$ & $18(9-29) / 18(8-29) / \mathrm{NA}$ & VP, CSI & 30d Mortality \\
\hline Wada 2013, Japan [21] & RS & $152(132)$ & $34(25-45) / 41(34-51) / \mathrm{NA}$ & CJ & 28d SMR (TRISS) \\
\hline Sierink 2013, The Netherlands [4] & RS & $301(151)$ & $22(18-27) / 25$ (17-29)/NA & VP, CSI & Radiation exposure \\
\hline Huber-Wagner 2013, Germany [11] & RS & $16,719(9233)$ & $30(12) / 28(12) / 29(12)^{\mathrm{a}}$ & Not defined & SMR (RISC) \\
\hline Sedlic 2013, Canada [13] & RS & $67(67)$ & NA & VP, TM, CSI & SMR (TRISS) \\
\hline Kimura 2013, Japan [22] & RS & $5208(1858)$ & $\begin{array}{l}26(25-26) / 23(23-24) / \\
\mathrm{NA}^{\mathrm{a}}\end{array}$ & VP: GCS & SMR (TRISS) \\
\hline Hsiao 2013, Australia [7] & RS & $660(98)$ & $17(16) / 5(6) / \mathrm{NA}^{\mathrm{a}}$ & CJ/PM: VP, TM, FTTA & Multi-region injured $^{\mathrm{c}}$ \\
\hline Asha 2012, Australia [3] & RS & $1280(624)$ & $4(2-10) / 4(2-10) / 4(2-10)$ & VP, TM/CJ, CSI & $\begin{array}{l}\text { Radiation exposure/ } \\
\text { missed injuries }\end{array}$ \\
\hline Babaud 2012, France [6] & PS & $339(189)$ & NA & VP, TM, CSI (Vittel) & Unsuspected injuries \\
\hline Stengel 2012, Germany [23] & RS & $982(982)$ & $25(18-33) /-/ 25(18-33)$ & $\begin{array}{l}\text { VP, TM, CSI, CJ } \\
\text { (DGU) }\end{array}$ & Missed injuries \\
\hline Hutter 2011, Germany [24] & OS & $1144(608)$ & $21(9) / 28(12) / \mathrm{NA}^{\mathrm{a}}$ & VP, TM & Mortality \\
\hline Gupta 2011, USA [17] & PS & $701(600)$ & $5(1-14) / 2(1-5) / 5(1-13)$ & CJ & Missed injuries \\
\hline Smith 2011, UK [25] & OS & $254(138)$ & $\begin{array}{l}14(11) / 7(6) / \mathrm{NA}^{\mathrm{a}} \\
13(11) / 7(9) / \mathrm{NA}\end{array}$ & $\mathrm{TM}$ & Change of treatment \\
\hline Wurmb 2011, Germany [26] & RS & $318(163)$ & $27(17-41) / 24(13-34) / \mathrm{NA}$ & $\begin{array}{l}\text { VP, TM, CSI (Nast- } \\
\text { Kolb) }\end{array}$ & $\begin{array}{l}\text { Time to surgery/mortal- } \\
\text { ity }\end{array}$ \\
\hline Smith 2012, UK [27] & Survey & 245 hospitals & - & VP, TM, CSI, PMI, WS & - \\
\hline Tillou 2009, USA [18] & PS & 284 & $13(1-17) /-/ 13(1-17)$ & $\mathrm{CJ}$ & Unsuspected injuries \\
\hline Huber-Wagner 2009, Germany [2] & RS & $4621(1494)$ & $32(14) / 28(12) / 30(13)^{\mathrm{a}}$ & Not defined & SMR (TRISS/RISC) \\
\hline Wurmb 2009, Germany [28] & RS & $161(82)$ & $24(11-33) / 22(11-32) / \mathrm{NA}$ & $\begin{array}{l}\text { VP, TM, CSI (Nast- } \\
\text { Kolb) }\end{array}$ & Time to diagnosis \\
\hline Rieger, 2009, Austria [16] & RS & 88 & $29(10) /-/ 29(10)^{\mathrm{a}}$ & $\begin{array}{l}\text { VP, TM, CSI (Nast- } \\
\text { Kolb) }\end{array}$ & $\begin{array}{l}\text { Time to diagnosis/missed } \\
\text { injuries }\end{array}$ \\
\hline Nguyen 2009, Swiss [29] & OS & 90 & NA & $\mathrm{TM}$ & Examination time \\
\hline Wurmb 2007, Germany [8] & RS & $120(85)$ & NA/NA/19 (3-75) & $\begin{array}{l}\text { VP, TM, CSI (Nast- } \\
\text { Kolb) }\end{array}$ & Polytrauma (ISS $\geq 16$ ) \\
\hline Weninger 2007, Austria [15] & OS & $370(185)$ & $27(10) / 28(12) / \mathrm{NA}^{\mathrm{a}}$ & Not defined & $\begin{array}{l}\text { Accuracy/time to diag- } \\
\text { nosis }\end{array}$ \\
\hline Prokop 2006, Germany [12] & RS & 100 & $33(12) /-/ 33(12)^{a}$ & $\mathrm{CJ}$ & Examination time \\
\hline Salim 2006, USA [19] & PS & 1000 & NA & $\begin{array}{l}\text { Normal abdominal PE, } \\
\text { and TM }\end{array}$ & Change of treatment \\
\hline Sampson 2006, UK [30] & $\mathrm{RS}$ & 296 & NA & Not defined & (unsuspected) injuries \\
\hline Wurmb 2005, Germany [31] & PC & $120(78)$ & NA & $\begin{array}{l}\text { VP, TM, CSI (Nast- } \\
\text { Kolb) }\end{array}$ & Examination time \\
\hline Heyer 2005, Germany [32] & $\mathrm{RCT}$ & 80 & NA & CJ & $\begin{array}{l}\text { Examination time/radia- } \\
\text { tion exposure }\end{array}$ \\
\hline Albrecht 2004, Germany [33] & RS & 50 & NA & CJ & Missed injuries \\
\hline Self 2003, USA [20] & $\mathrm{RC}$ & 457 & NA & CJ & Change of treatment \\
\hline Leidner 1998, Sweden [34] & PS & 111 & NA & CJ & $\begin{array}{l}\text { Examination time/missed } \\
\text { injuries }\end{array}$ \\
\hline
\end{tabular}

$I S S$ Injury Severity Score, $I Q R$ interquartile ranges, $C M$ case-matched study, $R S$ retrospective study, $P M$ prediction model, $O S$ observational study, $P S$ prospective study, $R C T$ randomized clinical trial, $V P$ vital parameters, $T M$ trauma mechanism, $C S I$ clinical suspicious injury, $C J$ clinical judgement, FTTA full trauma team activation, $P E$ physical examination, $S M R$ standardized mortality ratio

${ }^{\text {a }}$ Mean, SD

b Mean, $95 \% \mathrm{CI}$

${ }^{c}$ Multi-region injured defined by AIS $\geq 2$ in $\geq 2$ body regions (head/face, vertebral column, chest, abdomen/pelvis) 
Table 2 Overview of reported mortality and polytrauma proportion in populations selected for TBCT studies

\begin{tabular}{|c|c|c|c|}
\hline Author, study year, country & $\begin{array}{l}\text { Eligibility criteria besides blunt trauma, adult and } \\
\text { direct transfer }\end{array}$ & $\begin{array}{l}\text { Mortality }(\%) \\
\text { TBCT/control/overall }\end{array}$ & $\begin{array}{l}\text { Polytrauma, ISS } \geq 16(\%) \\
\text { TBCT/control/overall }\end{array}$ \\
\hline $\begin{array}{l}\text { Sierink 2014, The Netherlands } \\
\text { [14] }\end{array}$ & $\geq 1 \mathrm{VP}$ or CSI & $13.0 / 13.0 / 13.0(30 \mathrm{~d})$ & $63.2 / 63.2 / 63.2$ \\
\hline Wada 2013, Japan [21] & Requiring bleeding control & $18.1 / 80.0 / 26.3(28 \mathrm{~d})$ & $>75 />75 />75$ \\
\hline Sierink 2013, The Netherlands [4] & ISS $\geq 16$ and $\geq 1 \mathrm{VP}$ or CSI & $5.3 / 4.6 / 5.0(30 \mathrm{~d})$ & 100 (by protocol) \\
\hline $\begin{array}{l}\text { Huber-Wagner 2013, Germany } \\
\text { [11] }\end{array}$ & ISS $\geq 16$ & 17.4/21.4/19.2 (overall) & 100 (by protocol) \\
\hline Sedlic 2013, Canada [13] & $\begin{array}{l}\text { TBCT performed, and ISS } \geq 16 \text {, and } \geq 1 \mathrm{VP}, \mathrm{TM} \text { or } \\
\text { CSI }\end{array}$ & 14.9/-/- (ND) & 100 (by protocol) \\
\hline Kimura 2013, Japan [22] & GCS 3-12, SBP > 75 mmHg & $24 / 28 / 27$ (ND) & NA \\
\hline Hsiao 2013, Australia [7] & Trauma team activation and initial CT scan required & $3.1 / 1.2 / 1.5(\mathrm{ND})$ & $51.5 / 16.5 / 21.7$ \\
\hline Asha 2012, Australia [3] & Trauma team activation & NA & $17.5 / 18.5 / 18.0$ \\
\hline Babaud 2012, France [6] & $\geq 1$ Vittel criterion & NA & NA \\
\hline Stengel 2012, Germany [23] & $\geq 1 \mathrm{VP}, \mathrm{TM}$ or CSI, CJ & 7.1/-/7.1 (ND) & 36.7 \\
\hline Hutter 2011, Germany [24] & Admission to trauma center & 15/8/13 (overall) & $95.1 / 96.9 / 95.5$ \\
\hline Gupta 2011, USA [17] & Trauma team activation after blunt trauma & NA & $-/-/ 20$ \\
\hline Smith 2011, UK [25] & Suspicion on having multiple or serious injuries & 4.7 (ND) & NA \\
\hline Wurmb 2011, Germany [26] & $\begin{array}{l}\text { (suspected) Multiple trauma requiring emergency } \\
\text { surgery }\end{array}$ & $5.8 / 5.5 / 5.7(30 \mathrm{~d})$ & $87.1 / 71.6 / 84.4$ \\
\hline Smith 2012, UK [27] & - & - & - \\
\hline Tillou 2009, USA [18] & Trauma team activation after blunt trauma & NA & NA \\
\hline Huber-Wagner 2009, Germany [2] & ISS $\geq 16$ & $21 / 22 / 22$ (overall) & 100 (by protocol) \\
\hline Wurmb 2009, Germany [28] & ISS $\geq 18$ & NA & 100 (by protocol) \\
\hline Rieger, 2009, Austria [16] & Treatment in resuscitation area by trauma team & NA & $67.0 / 58.2 / 62.7$ \\
\hline Nguyen 2009, Swiss [29] & TBCT performed, and MVC or fall from $>3 \mathrm{~m}$ & NA & NA \\
\hline Wurmb 2007, Germany [8] & Sedated and ventilated trauma patients & NA & $69.4 / 5.7 / 50.8$ \\
\hline Weninger 2007, Austria [15] & $\begin{array}{l}\text { ISS } \geq 17, \text { and AIS } \geq 4 \text { in } \geq 1 \text { body region (head, tho- } \\
\text { rax or abdomen), and survival until ICU admission }\end{array}$ & $17 / 16 / 17$ & 100 (by protocol) \\
\hline Prokop 2006, Germany [12] & ISS $>16$ and TBCT performed & $13 /-/ 13$ & 100 (by protocol) \\
\hline Salim 2006, USA [19] & $\begin{array}{l}\text { No visible evidence of chest or abdominal injury, } \\
\text { and hemodynamically stable, and PE of abdomen } \\
\text { normal or unevaluable because of depressed level } \\
\text { of consciousness, and significant mechanism of } \\
\text { injury }\end{array}$ & NA & NA \\
\hline Sampson 2006, UK [30] & $\begin{array}{l}\text { Hemodynamically stable, and AIS } \geq 2 \text { in } \geq 1 \text { body } \\
\text { region (head/neck, thorax, abdomen/pelvis, spine } \\
\text { or extremities) }\end{array}$ & NA & NA \\
\hline Wurmb 2005, Germany [31] & Treatment in resuscitation area by trauma team & NA & NA \\
\hline Heyer 2005, Germany [32] & $\begin{array}{l}\text { Suspected injury of } \geq 2 \text { body regions of which } \geq 1 \text { is } \\
\text { life-threatening, and ICU admission }\end{array}$ & NA & NA \\
\hline Albrecht 2004, Germany [33] & $\begin{array}{l}\text { Prehospital suspected polytrauma, and TBCT } \\
\text { performed }\end{array}$ & NA & NA \\
\hline Self 2003, USA [20] & Blunt head injury and TBCT performed & NA & NA \\
\hline Leidner 1998, Sweden [34] & $\begin{array}{l}\text { Hemodynamically stable, and clinical suspicion of } \\
\text { multiple organ injuries or a trauma mechanism } \\
\text { capable of producing major injury to multiple } \\
\text { organ systems. }\end{array}$ & NA & NA \\
\hline
\end{tabular}

ISS Injury Severity Score, $V P$ vital parameters, $T M$ trauma mechanism, $C S I$ clinical suspicious injury, $C J$ clinical judgement, $N D$ not defined, $N A$ not available 


\section{Discussion}

In this systematic review of studies that evaluate or describe indications for TBCT in initial trauma care, we showed similarities and differences of these indications. There is a wide variety of eligibility criteria and outcome measures between studies (Table 2). Combinations of compromised vital parameters, severe trauma mechanisms and clinical suspicion on severe injuries are most often reported, however, clinical judgement on expected severe and multiple injuries is described as well. Within these groups of indications, there is a large variation in used parameters and cutoff values (Supplementary Appendix 1). Because of this variety between sets of indications, it is difficult to compare indications for TBCT between studies.

Differences in outcome measures for justification of TBCT in hindsight implicate a lack of consensus toward patient groups that rightfully received a TBCT during their trauma work-up. Anatomical scoring systems with different thresholds for ISS and AIS for body regions are used to justify the performance of TBCT or to select patients who might benefit from TBCT scanning [7, 8]. Several retrospective studies on TBCT select patients by anatomical scoring systems and, therefore, suggest that patients above these thresholds could benefit from TBCT. Other outcome measures reflecting the severity or extent of injuries might be suitable as well, such as mortality, morbidity, ICU admittance, surgical and radiological interventions or detection of unsuspected injuries.

Not only parameters reflecting severe injury could justify TBCT. Decreased levels of consciousness could be considered an indication on itself since clinical indicators for imaging are unreliable owing to the lack of subjective input from the patient. Routine CT imaging for patients with unreliable physical examination is reported to reveal unsuspected findings in up to $38 \%$, leading to treatment changes in 19-26\% [19, 20]. Furthermore, one could hypothesize that TBCT might lead to early discharge for less severely injured patients when used to rule out injuries [19]. Since the probability of detecting injuries after major trauma during the clinical course of alert patients might be lowered after TBCT, the in-hospital observation of the clinical course might be less valuable.

This review included only three studies for which the main objective was to evaluate indications for TBCT in trauma patients. Studies that described mortality and ISS already chose study eligibility criteria to select patients that might benefit from TBCT. Thereby, the wide variety of eligibility criteria made comparison of mortality and ISS of patient groups selected for TBCT less valuable. Besides limited comparability of methods, there was also a low availability of mortality and ISS for the included studies.

An anatomical scoring system such as ISS as indication for TBCT cannot be used in daily practice, because the results are calculated after radiologic imaging is performed. As well as other outcome parameters reflecting severe injury, anatomical scoring systems could only be helpful as an outcome measure for the evaluation of the indication for TBCT and not to define the indication for TBCT.

In this overview of TBCT indications, we did not make a distinction between immediate TBCT and TBCT after conventional $\mathrm{X}$-rays and sonography. Future prospective research on the indication for one or both strategies should consider this difference in its design. Furthermore, there was no distinction made regarding different imaging protocols. Contrast enhancement and body position were not described for included studies.

Little is known of the predictive value of specific parameters within the sets of indications for severe and multiple injury. However, reduced Glasgow Coma Scale (GCS) after major trauma seems to be a valid indication for TBCT. First, it is reported to independently predict multi-region injury and detection of injury, in general. Second, the unreliability of the physical examination can result in unsuspected findings needing treatment. Decision for a cut-off value for GCS might depend on which goal one pursues: to select multiple and severely injured patients or reduction of missed injuries after major trauma.

Future research needs to prospectively determine the positive predictive value of separate TBCT indications for multiple and severely injured patients. Positive predictive values for TBCT indications are useful for determining the proportion of patients that were appropriately selected for TBCT, and the concomitant radiation exposure could, therefore, be accepted. To determine the proportion of the multiple and severely injured patients selected for TBCT, sensitivity of a set of indications has to be calculated. Emphasis on specific diagnostic tests changes when another type of outcome measure is chosen such as reduction of missed injuries.

The question remains as to whether we should use fixed sets of indications for TBCT, and, if so, how they should be defined. In the meantime, one should be aware that selection of patients for TBCT by clinical judgement alone could result in relatively low ISS. Independently from which outcome measure is chosen, one should carefully weigh the potential benefits of TBCT to an increased radiation exposure and potential increase of costs. The unsuspected findings and eventual shortening of hospital admission should outweigh the increased radiation exposure to make TBCT beneficial for the less severely injured patients. 


\section{Conclusion}

Indications for TBCT in trauma show a wide variety in formulation and cut-off values for vital parameters and trauma mechanism dimensions. Combinations of compromised vital parameters, severe trauma mechanisms and clinical suspicion on severe injuries are often used. However, clinical judgement on expected severe and multiple injury is used as well. Consensus on outcome measures for justification of TBCT should be obtained to guide further research on the appropriate indications for TBCT in trauma.

Acknowledgments The authors thank J. G. Daams (clinical librarian, Academic Medical Center, Amsterdam, the Netherlands) for his support in conducting the search of the literature.

\section{Compliance with ethical standards}

Conflict of interest Kaij Treskes, Teun Peter Saltzherr, Jan Luitse, Ludo Beenen, and Carel Goslings declare that they have no conflict of interest.

Ethical standard This article does not contain any studies with human participants or animals performed by any of the authors.

Open Access This article is distributed under the terms of the Creative Commons Attribution 4.0 International License (http://creativecommons.org/licenses/by/4.0/), which permits unrestricted use, distribution, and reproduction in any medium, provided you give appropriate credit to the original author(s) and the source, provide a link to the Creative Commons license, and indicate if changes were made.

\section{References}

1. American College of Surgeons Committee on Trauma: ATLS advanced trauma life support program for doctors. Chicago 2013.

2. Huber-Wagner S, Lefering R, Qvick LM, Korner M, Kay MV, Pfeifer KJ, et al. Effect of whole-body CT during trauma resuscitation on survival: a retrospective, multicentre study. Lancet. 2009;373(9673):1455-61.

3. Asha S, Curtis KA, Grant N, Taylor C, Lo S, Smart R, et al. Comparison of radiation exposure of trauma patients from diagnostic radiology procedures before and after the introduction of a panscan protocol. Emerg Med Australas. 2012;24(1):43-51.

4. Sierink JC, Saltzherr TP, Wirtz MR, Streekstra GJ, Beenen LF, Goslings JC. Radiation exposure before and after the introduction of a dedicated total-body CT protocol in multitrauma patients. Emerg Radiol. 2013;20(6):507-12.

5. Stengel D, Frank M, Matthes G, Schmucker U, Seifert J, Mutze S, et al. Primary pan-computed tomography for blunt multiple trauma: can the whole be better than its parts? Injury. 2009;40(Suppl 4):S36-46.

6. Babaud J, Ridereau-Zins C, Bouhours G, Lebigot J, Le GR, Bertrais $\mathrm{S}$, et al. Benefit of the Vittel criteria to determine the need for whole body scanning in a severe trauma patient. Diagn Interv Imaging. 2012;93(5):371-9.

7. Hsiao KH, Dinh MM, McNamara KP, Bein KJ, Roncal S, Saade $\mathrm{C}$, et al. Whole-body computed tomography in the initial assessment of trauma patients: is there optimal criteria for patient selection? Emerg Med Australas. 2013;25(2):182-91.
8. Wurmb TE, Fruhwald P, Hopfner W, Roewer N, Brederlau J. Whole-body multislice computed tomography as the primary and sole diagnostic tool in patients with blunt trauma: searching for its appropriate indication. Am J Emerg Med. 2007;25(9):1057-62.

9. Moher D, Liberati A, Tetzlaff J, Altman DG. Preferred reporting items for systematic reviews and meta-analyses: the PRISMA statement. BMJ. 2009;339:b2535.

10. Riou B, Thicoïpé M, Atain-Kouadio P, Carli P. Le traumatisé grave. Actualités en réanimation préhospitalière. Journées scientifiques des SAMU de France. 2002.

11. Huber-Wagner S, Biberthaler P, Haberle S, Wierer M, Dobritz $\mathrm{M}$, Rummeny E, et al. Whole-body CT in haemodynamically unstable severely injured patients-a retrospective, multicentre study. PLoS One. 2013;8(7):e68880.

12. Prokop A, Hotte H, Kruger K, Rehm KE, Isenberg J, Schiffer G. Multislice CT in diagnostic work-up of polytrauma. Unfallchirurg. 2006;109(7):545-50.

13. Sedlic A, Chingkoe CM, Tso DK, Galea-Soler S, Nicolaou S. Rapid imaging protocol in trauma: a whole-body dual-source CT scan. Emerg Radiol. 2013;20(5):401-8.

14. Sierink JC, Saltzherr TP, Beenen LF, Russchen MJ, Luitse JS, Dijkgraaf MG, et al. A case-matched series of immediate totalbody CT scanning versus the standard radiological work-up in trauma patients. World J Surg. 2014;38(4):795-802.

15. Weninger P, Mauritz W, Fridrich P, Spitaler R, Figl M, Kern $\mathrm{B}$, et al. Emergency room management of patients with blunt major trauma: evaluation of the multislice computed tomography protocol exemplified by an urban trauma center. J Trauma. 2007;62(3):584-91.

16. Rieger M, Czermak B, El AR, Sumann G, Jaschke W, Freund M. Initial clinical experience with a 64-MDCT whole-body scanner in an emergency department: better time management and diagnostic quality? J Trauma. 2009;66(3):648-57.

17. Gupta M, Schriger DL, Hiatt JR, Cryer HG, Tillou A, Hoffman $\mathrm{JR}$, et al. Selective use of computed tomography compared with routine whole body imaging in patients with blunt trauma. Ann Emerg Med. 2011;58(5):407-16.

18. Tillou A, Gupta M, Baraff LJ, Schriger DL, Hoffman JR, Hiatt $\mathrm{JR}$, et al. Is the use of pan-computed tomography for blunt trauma justified? A prospective evaluation. J Trauma. 2009;67(4):779-87.

19. Salim A, Sangthong B, Martin M, Brown C, Plurad D, Demetriades D. Whole body imaging in blunt multisystem trauma patients without obvious signs of injury: results of a prospective study. Arch Surg. 2006;141(5):468-73.

20. Self ML, Blake AM, Whitley M, Nadalo L, Dunn E. The benefit of routine thoracic, abdominal, and pelvic computed tomography to evaluate trauma patients with closed head injuries. Am J Surg. 2003;186(6):609-13.

21. Wada D, Nakamori Y, Yamakawa K, Yoshikawa Y, Kiguchi T, Tasaki $\mathrm{O}$, et al. Impact on survival of whole-body computed tomography before emergency bleeding control in patients with severe blunt trauma. Crit Care. 2013;17(4):R178.

22. Kimura A, Tanaka N. Whole-body computed tomography is associated with decreased mortality in blunt trauma patients with moderate-to-severe consciousness disturbance: a multicenter, retrospective study. J Trauma Acute Care Surg. 2013;75(2):202-6.

23. Stengel D, Ottersbach C, Matthes G, Weigeldt M, Grundei S, Rademacher G, et al. Accuracy of single-pass whole-body computed tomography for detection of injuries in patients with major blunt trauma. CMAJ. 2012;184(8):869-76.

24. Hutter M, Woltmann A, Hierholzer C, Gartner C, Buhren V, Stengel D. Association between a single-pass whole-body computed tomography policy and survival after blunt major trauma: a retrospective cohort study. Scand J Trauma Resusc Emerg Med. 2011;19:73. 
25. Smith CM, Woolrich-Burt L, Wellings R, Costa ML. Major trauma CT scanning: the experience of a regional trauma centre in the UK. Emerg Med J. 2011;28(5):378-82.

26. Wurmb TE, Quaisser C, Balling H, Kredel M, Muellenbach R, Kenn W, et al. Whole-body multislice computed tomography (MSCT) improves trauma care in patients requiring surgery after multiple trauma. Emerg Med J. 2011;28(4):300-4.

27. Smith CM, Mason S. The use of whole-body CT for trauma patients: survey of UK emergency departments. Emerg Med J. 2012;29(8):630-4.

28. Wurmb TE, Fruhwald P, Hopfner W, Keil T, Kredel M, Brederlau J, et al. Whole-body multislice computed tomography as the first line diagnostic tool in patients with multiple injuries: the focus on time. J Trauma. 2009;66(3):658-65.

29. Nguyen D, Platon A, Shanmuganathan K, Mirvis SE, Becker $\mathrm{CD}$, Poletti PA. Evaluation of a single-pass continuous wholebody 16-MDCT protocol for patients with polytrauma. AJR Am J Roentgenol. 2009;192(1):3-10.
30. Sampson MA, Colquhoun KB, Hennessy NL. Computed tomography whole body imaging in multi-trauma: 7 years experience. Clin Radiol. 2006;61(4):365-9.

31. Wurmb T, Fruhwald P, Brederlau J, Steinhubel B, Frommer M, Kuhnigk $\mathrm{H}$, et al. The Wurzburg polytrauma algorithm. Concept and first results of a sliding-gantry-based computer tomography diagnostic system. Anaesthesist. 2005;54(8):763-8.

32. Heyer CM, Rduch G, Kagel T, Lemburg SP, Theisinger A, Bauer TT, et al. Prospective randomized trial of a modified standard multislice CT protocol for the evaluation of multiple trauma patients. Rofo. 2005;177(2):242-9.

33. Albrecht T, von Schlippenbach J, Stahel PF, Ertel W, Wolf KJ. The role of whole body spiral CT in the primary work-up of polytrauma patients - comparison with conventional radiography and abdominal sonography. Rofo. 2004;176(8):1142-50.

34. Leidner B, Adiels M, Aspelin P, Gullstrand P, Wallen S. Standardized CT examination of the multitraumatized patient. Eur Radiol. 1998;8(9):1630-8. 